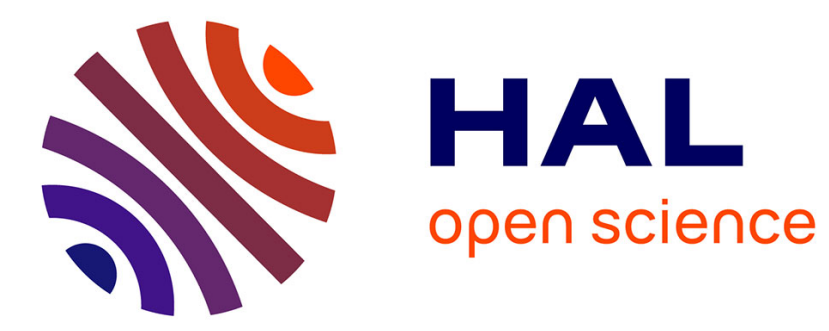

\title{
Phosphahelicenes with (Thio)Phosphinic Acid and Ester Functions by the Oxidative Photocyclisation Approach
}

\author{
Julie Febvay, Charles Demmer, Pascal Retailleau, Jeanne Crassous, Laura
}

Abella, Jochen Autschbach, Arnaud Voituriez, Angela Marinetti

\section{- To cite this version:}

Julie Febvay, Charles Demmer, Pascal Retailleau, Jeanne Crassous, Laura Abella, et al.. Phosphahelicenes with (Thio)Phosphinic Acid and Ester Functions by the Oxidative Photocyclisation Approach. Chemistry - A European Journal, 2019, 25 (68), pp.15609-15614. 10.1002/chem.201903750 . hal02377389

\section{HAL Id: hal-02377389 \\ https://hal.science/hal-02377389}

Submitted on 9 Dec 2019

HAL is a multi-disciplinary open access archive for the deposit and dissemination of scientific research documents, whether they are published or not. The documents may come from teaching and research institutions in France or abroad, or from public or private research centers.
L'archive ouverte pluridisciplinaire HAL, est destinée au dépôt et à la diffusion de documents scientifiques de niveau recherche, publiés ou non, émanant des établissements d'enseignement et de recherche français ou étrangers, des laboratoires publics ou privés. 


\title{
Phosphahelicenes with (thio)phosphinic acid and ester functions via the oxidative photocyclisation approach
}

\author{
Julie Febvay, ${ }^{[a]}$ Charles S. Demmer, ${ }^{[a]}$ Pascal Retailleau, ${ }^{[a]}$ Jeanne Crassous, ${ }^{[c]}$ Laura Abella, ${ }^{[b]}$ Jochen \\ Autschbach, ${ }^{*[b]}$ Arnaud Voituriez ${ }^{*[a]}$ and Angela Marinetti; ${ }^{*[a]}$
}

\begin{abstract}
Phosphahelicenes with thiophosphinic acid and ester functions have been obtained via the oxidative photocyclisation of olefins bearing both a benzophenanthrene and a benzophosphole unit. When the method has been extended to olefins bearing a partially saturated benzophospholene unit, a divergent regioselectivity of the photocyclisation step has been observed, leading to new helicenes where the phosphorus function is located on the external rim of the helical backbone. The observed regioselectivity correlates well with the free-valence numbers of the atoms involved in the photocyclisation reaction (DFT calculations).
\end{abstract}

\section{Introduction}

Although helicenes have attracted attention for more than one hundred years now, their chemistry still represents a hot topic, due to their peculiar physicochemical and optoelectronic properties and applications, as well as to the chirality related phenomena displayed by these inherently chiral polyaromatic compounds. ${ }^{[1]}$ A common strategy to broaden and modulate the structural features and properties of helicenes is the incorporation of heterocyclic units in their backbones. ${ }^{[1]]}$ Thus, for instance, nitrogen and phosphorus heterocycles have been incorporated, which opened the way, notably, to new applications in coordination chemistry, organometallic catalysis and organocatalysis. ${ }^{[2]}$ In the phosphahelicene series, compounds with phosphine oxides and sulfide functions, trivalent phosphines and the corresponding metal complexes, have been extensively investigated by our group and others. ${ }^{[2 b}$, 3] Representative examples are displayed in Scheme 1. Phosphine oxides have been prepared either by building the phosphorus ring at a late stage $^{[4]}$ or from preformed, suitably functionalized benzophosphole oxides. ${ }^{[5]}$ The same strategies have been

[a] J. Febvay, Dr. C. Demmer, Dr. P. Retailleau, Dr. A. Voituriez ${ }^{*}$ and Dr. A. Marinetti*

Institut de Chimie des Substances Naturelles, CNRS UPR 2301 Université Paris-Sud, Université Paris-Saclay

1, av. de la Terrasse, 91198 Gif-sur-Yvette

E-mail: angela.marinetti@cnrs.fr arnaud.voituriez@cnrs.fr

[b] Dr. L. Abella, Prof. J. Autschbach

Department of Chemistry

University at Buffalo, State University of New York

Buffalo, NY 14260-3000 (USA)

E-mail: jochena@buffalo.edu

[c] Dr. J. Crassous

Univ Rennes, Institut des Sciences Chimiques de Rennes UMR CNRS 6226, Campus de Beaulieu, 35042 Rennes Cedex, France Supporting information for this article is given via a link at the end of the document. applied occasionally to the synthesis of phosphahelicenes with phosphinic acid, ${ }^{[6]}$ phosphinic esters ${ }^{[4 a, 7]}$ and phosphinamide functions, ${ }^{[6]}$ but these classes of compounds have been investigated only briefly. Thus, phosphahelicenes displaying $P(V)$ functions represent an almost untouched domain for future applications, that is obviously conditional on the development of suitable synthetic approaches.

Here we report on our investigations toward the synthesis of new helical phosphinates and phosphinic acids and we describe the first phosphahelicenes displaying thiophosphinic acid functions $\left(\mathrm{R}_{2} \mathrm{P}(\mathrm{S}) \mathrm{OH}\right)$. We also demonstrate here a suitable access to the first helicenes displaying terminal phospholene rings, instead of the usual phosphole units.

One relevant feature of most of these compounds is to associate a helically chiral scaffold with a configurationally stable, stereogenic phosphorus centre. This might result in matchmismatch effects on their chirality-related properties with potential interest in a variety of fields including enantioselective catalysis ${ }^{[2 \mathrm{~b}}$ ${ }^{3,8]}$ and optoelectronic material sciences. ${ }^{[4 \mathrm{~d}, 7,9]}$

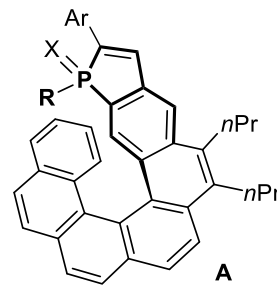
$\mathrm{X}=\mathrm{O}, \mathrm{S}$, lone pair

A2: $\mathrm{R}=\mathrm{OH}, \mathrm{NH}_{2}$ $\mathrm{X}=\mathrm{O}$
A1: $R=$ alkyl, aryl

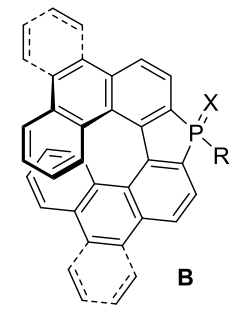

B1: $\mathrm{R}=\mathrm{Ph}$,

$X=O, S$, lone pair

B2: $R=O M e, X=O$

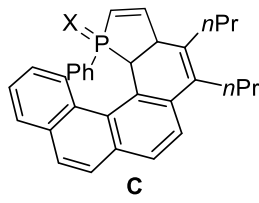

$\mathrm{X}=\mathrm{O}, \mathrm{S}$, lone pair

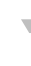

Scheme 1 Representative phosphahelicenes from the literature.

\section{Results and Discussion}

\section{Helicenes with terminal phosphole units}

The synthetic studies above rely on the well-known Mallory oxidative photocyclisations of diarylolefins ${ }^{[10]}$ to create the helical backbones of (thio)phosphinic acids and esters. The first part of our work focused on the synthesis of the thiophosphinic ester 3 (Scheme 2, $R=M e$ ) and the corresponding acid $(R=H)$, that are structural analogues of the phosphinic acid $\mathbf{A 2}(\mathrm{R}=\mathrm{OH}, \mathrm{X}=$ O) already reported by our group. ${ }^{[6]}$ The retrosynthetic approach envisioned first, involves the oxidative photocyclization of olefin 2 as the key step (Scheme 2, path II). Olefin 2 combines a benzophenanthrene and a P-methoxy thiophosphindole units. It might be obtained from either the P-methoxy thiophosphindole 1 via coupling reactions (path I), or by sulfuration of the preformed 
WILEY-VCH

olefinic phosphinate $2^{\text {;[6] }}$ (path II). Alternatively, the helical thiophosphinic ester $3(\mathrm{R}=\mathrm{Me})$ might be obtained by sulfuration of the corresponding helical phosphinite 3' (path III). In this work, we have investigated the three strategies, for comparison purposes.

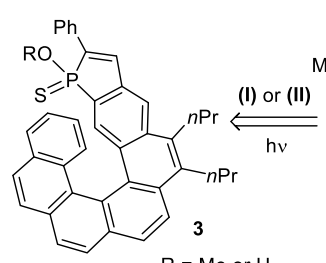

(III) $\mathrm{R}=\mathrm{Me}$ or $\mathrm{H}$

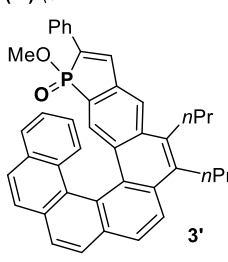

Scheme 2. Retrosynthetic approaches to 3 via the oxidative photocyclization method: paths (I), (II) and (III)

[Note: all throughout this manuscript, compounds with the same molecular structure, but displaying $P(S)$ and $P(O)$ functions have been numbered $N$ and $N$ ' respectively].

Considering path I in Scheme 2, the starting P-methoxy-2phenylphosphindole sulfide 1 could be obtained easily by sulfuration of the corresponding phosphinate with the Lawesson reagent $(90 \%$ isolated yield, Scheme 3a), however the subsequent Suzuki coupling of $\mathbf{1}$ with the olefinic dioxaborolane shown in Scheme 3, could not be achieved in satisfactory yields. In parallel experiments, following path II in Scheme 2, O/S exchange on the olefinic methyl phosphinate 2' was shown to take place in good yield (73\%), leading to the desired olefin 2 in a straightforward way (Scheme 3b).

(a)

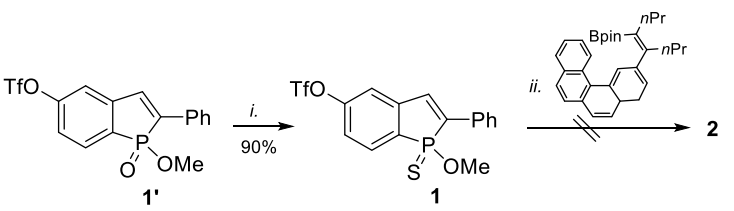

(b)

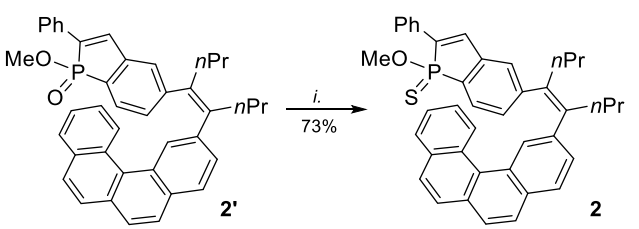

i. Lawesson reagent $\left(0.8\right.$ eq.), toluene, $90^{\circ} \mathrm{C}, 4 \mathrm{~h}$

ii. $\mathrm{Pd}\left(\mathrm{PPh}_{3}\right)_{4}(10 \mathrm{~mol} \%), \mathrm{K}_{2} \mathrm{CO}_{3}$ (3 equiv), THF/ $\mathrm{H}_{2} \mathrm{O}: 10 / 1,80^{\circ} \mathrm{C}$ Scheme 3 Synthesis of olefin 2 displaying a thiophosphinate function.

Next, the olefinic thiophosphinate $\mathbf{2}$ as well as the methyl phosphinate 2' have been subjected to oxidative photocyclization reactions in the presence of an excess iodine and propylene oxide (Scheme $4 \mathrm{a}$ and $4 \mathrm{~b}$ respectively). These reactions gave the desired helical thiophosphinate $\mathbf{3}$ and phosphinate 3', respectively. In both reactions, only one out of four possible cyclization products was isolated in significant amount. These photocyclizations selectively involve the $\mathrm{C} 1$ carbon of the benzophenanthrene unit and the $\mathrm{C} 6$ carbon of the benzophospholene unit, leading to $\mathbf{3}$ and $\mathbf{3}^{\prime}$ ' in $35 \%$ conversion and $60 \%$ yield, respectively. The photocyclisation of the phosphinate 2' led to 3' as a 2:1 mixture of diastereomers, with opposite relative configurations $\left(P^{*}, R_{\mathrm{P}}{ }^{*}\right)$ and $\left(M^{*}, R_{\mathrm{P}}{ }^{*}\right)$ of the two stereogenic units, namely the helical scaffold and the phosphorus centre. The two diastereoisomers of 3' could not be separated and therefore a definite structural assignment could not be achieved at this point. It could be achieved however by converting the methyl phosphinate $3^{\prime}$ into the phosphinic acid $4^{[6]}$ in quantitative yield, by reaction with DABCO (Scheme 4). The acid 4 has been unambiguously characterized by X-ray diffraction studies. Its solid-state structure is displayed in Scheme 4 (ORTEP view).

(a)<smiles>COP1(=S)Oc2ccc(C(=C(C(C)C)C(C)C)C(C)C)cc2C=C1c1ccccc1</smiles>

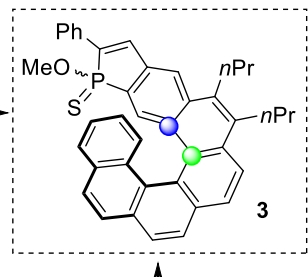

(b)
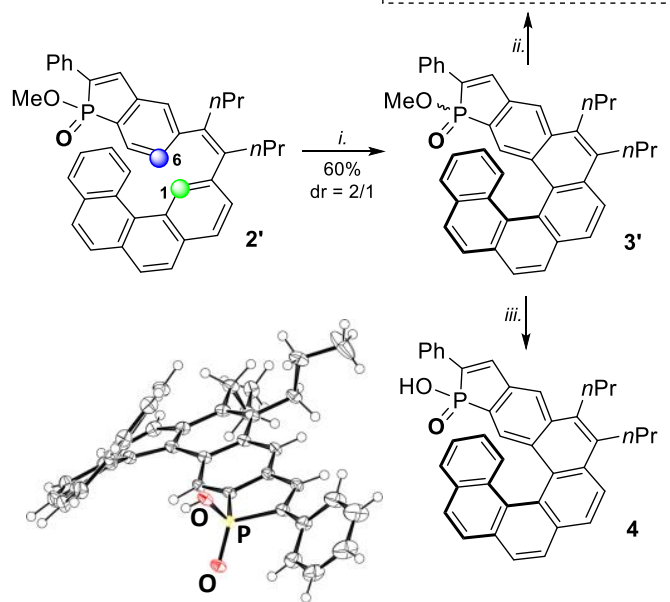

i. hv, $\mathrm{I}_{2}$, propylene oxide, $\left(\mathrm{ClCH}_{2}\right)_{2}, 45 \mathrm{~min}$

ii. Lawesson reagent ( 0.8 equiv.), toluene, $90^{\circ} \mathrm{C}, 4 \mathrm{~h}$ iii. $\mathrm{DABCO}, \mathrm{CH}_{3} \mathrm{CN}, 80^{\circ} \mathrm{C}, 16 \mathrm{~h}$

Scheme 4. Two alternative pathways $(a, b)$ for the synthesis of the helical methyl thiophosphinate 3 . And X-ray crystal structure of the phosphinic acid 4 Note: In compound 4, the phosphorus center is not stereogenic, due to $\mathrm{H}$-shift between the two oxygens.

The thiophosphinate 3 has been characterized by ${ }^{31} \mathrm{P}$ and mass spectrometry only, because of its low solubility in most solvents. Nevertheless, its structural assignment has been confirmed by showing that the same compound, $\mathbf{3}$, can be obtained through sulfuration of phosphinate 3' (Scheme 4). The quantitative O/S exchange reaction led to 3 as a mixture of isomers in the same $2: 1$ ratio as for the starting material (see supporting information). Overall, these experiments have enabled two alternative synthetic approaches to compound $\mathbf{3}$, which is the first phosphahelicene with a thiophosphinate function. 


\section{Helicenes with terminal phospholene units}

Additional studies have been focused then on the synthesis of helical (thio)phosphinates and (thio)phosphinic acids displaying phospholene units as the terminal rings. According to our general strategy, the synthetic approach makes use of suitably functionalized benzophospholenes (5 or $\mathbf{5}^{\prime}$ in Scheme 5 ) as building blocks that will be incorporated into the helical backbones In order to access these substrates, we have sought conditions for the selective hydrogenation of the olefinic double bond of the $\mathrm{P}-\mathrm{MeO}-$ phosphindole oxide 1'. Initial attempts carried out with $\mathrm{PtO}_{2} / \mathrm{H}_{2}$, L-selectride and $\mathrm{NiCl}_{2} / \mathrm{NaBH}_{4}$ were ineffective, as they led to either recovery of the starting material or to decomposition. Finally, hydrogenation in the presence of $10 \% \mathrm{Pd} / \mathrm{C}^{[11]}$ allowed obtention of the desired benzophospholene $\mathbf{5}^{\prime}$.

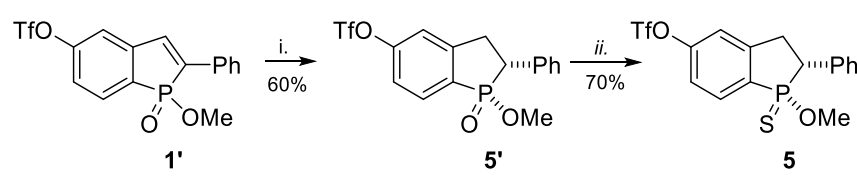

i. $\mathrm{H}_{2}, \mathrm{Pd} / \mathrm{C} 10 \%$, Heptane/AcOEt : $1 / 1$, r.t., $3 \mathrm{~h}$

ii. Lawesson reagent, toluene, reflux, $2 \mathrm{~h}$

Scheme 5 Synthesis of 5 via chemo- and diastereoselective hydrogenation of 1 ' and subsequent $\mathrm{O} / \mathrm{S}$ exchange.

Most rewardingly, the benzophospholene $\mathbf{5}^{\text {' }}$ was isolated as a single isomer, while the hydrogenations of other phospholene oxides, under analogous conditions, are known to produce mixtures of isomers, ${ }^{[12]}$ unless additional substituents hinder one of the faces of the phospholene. ${ }^{[13]}$ The stereochemistry of the hydrogenation reaction in Scheme 5 could be established from the X-ray crystal structure of the thiophosphinate $\mathbf{5}$ obtained from

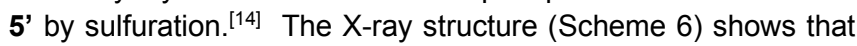
hydrogenation of 1' takes place from the face opposite to the OMe substituent on phosphorus, since the phenyl and OMe groups are in a relative syn-configuration in the product.

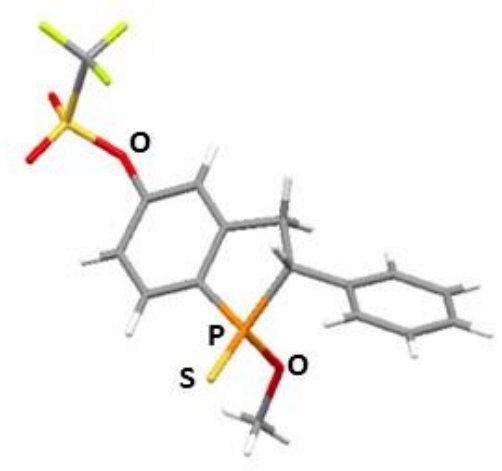

Scheme 6 X-Ray crystal structure of the 1-methoxy-2-phenylbenzophospholene sulphide 5 .

It may be mentioned here that treatment of the thiophosphinate $\mathbf{5}$ with $\mathrm{DABCO}$ leads to the corresponding thiophosphinic acid whose structure has been confirmed also by X-ray diffraction studies (for experimental details and X-ray data, see S.I.).

The benzophospholene oxide 5' has been used to build helicenes with terminal phospholene units, by following the two step path shown in Scheme 7. The triflate function of 5' enables a $\mathrm{Pd}(0)$ promoted Suzuki coupling with the benzophenanthrene substituted vinylboronate (62\% yield). The resulting olefin $\mathbf{6}^{\prime}$ ' has been subjected then to the oxidative photocyclization reaction in the presence of iodine and propylene oxide. The photocyclization takes place easily, with total conversion after $45 \mathrm{~min}$ only, but unexpectedly, it involves the C4 carbon of the benzophospholene unit, instead of the $\mathrm{C} 6$ carbon. It affords indeed the [7]helicenes 7'a/7'b as the only isolated products. The product consists in a 2:1 mixture of diastereomers with opposite configurations of the helical scaffold with respect to the stereogenic phosphorus and carbon centres ( ${ }^{31} \mathrm{P}$ NMR: $\delta=55.4 \mathrm{ppm}$ for the major isomer, $\delta=$ $59.0 \mathrm{ppm}$ for the minor isomer). The isomers could be separated by column chromatography on silica gel and fully characterized. Their molecular structures have been assigned based on NMR data. Thus, for instance, the ${ }^{1} \mathrm{H}$ NMR spectrum of the major isomer 7'a shows diagnostic doublets of doublets for $H_{a}$ and $H_{b}$ at $\delta=7.67\left(\mathrm{t}, J_{H-H}=J_{H-P}=8,4 \mathrm{~Hz}\right)$ and $\delta=8.20\left(\mathrm{dd}, J_{H-H}=8.4 \mathrm{~Hz}\right.$, $J_{H-P}=3.2 \mathrm{~Hz}$ ) ppm. Their stereochemistry has been assigned by chemical correlation with the corresponding thiophosphinic esters 7a,b (see Scheme 8 and Figure 1 hereafter and SI).

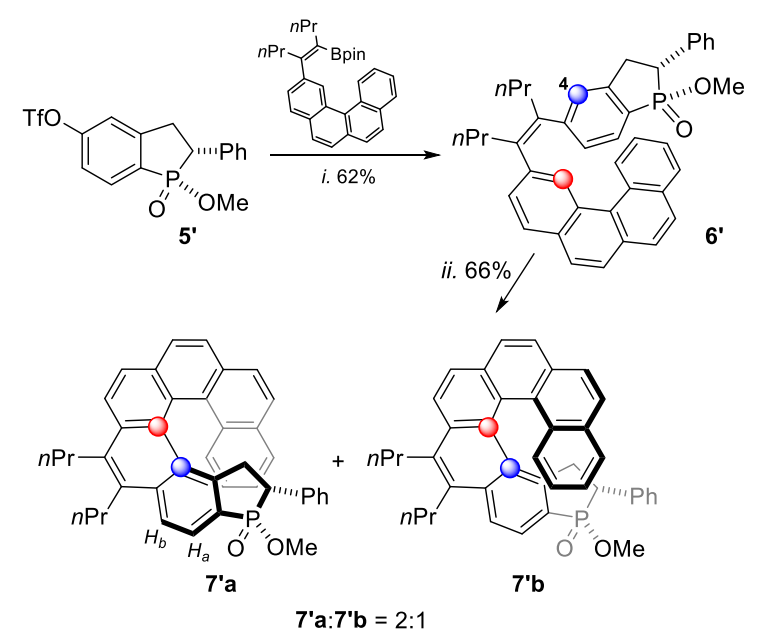

i. $\mathrm{Pd}\left(\mathrm{PPh}_{3}\right)_{4}(5 \mathrm{~mol} \%), \mathrm{Cs}_{2} \mathrm{CO}_{3}$ (3 equiv), $\mathrm{THF} / \mathrm{H}_{2} \mathrm{O}: 10 / 1,60^{\circ} \mathrm{C}, 16 \mathrm{~h}$ ii. $h v, \mathrm{I}_{2}$, propylene oxide, $\left(\mathrm{ClCH}_{2}\right)_{2}, 45 \mathrm{~min}$

Scheme 7. Synthesis of 7 ' via regioselective oxidative photocyclization of the phosphinate 6'.

The regioselectivity of this reaction sharply contrasts with the regioselectivity of the photocyclizations shown in Scheme 4, as well as with that of the analogous reactions performed so far on other benzophosphole derivatives (phosphinic acids, ${ }^{[6]}$, phosphole oxides $\left.{ }^{[5 a-c]}\right)$. In all these instances, the major and perhaps unique reaction pathway was the cyclization on the $\mathrm{C} 6$ carbon of the phosphindole unit.

Generally speaking, factors that control the regioselectivity of the $6 \pi$-electrocyclisations of this class are still poorly understood. In initial as well as in more recent studies, the regioselectivity has been tentatively correlated to various parameters, including the sum of the free-valence numbers of the atoms involved in the cyclization step $\left(\Sigma \mathrm{F}_{\mathrm{r}, \mathrm{s}}{ }^{*}\right) \cdot{ }^{[15]}$ This method has been considered here to rationalize the observed reaction pathways. These studies are summarized in the last section of this manuscript. 
WILEY-VCH

Given this unusual behavior of the $P$-methoxy benzophospholene oxide $6^{\prime}$ in the oxidative photocyclization reaction, for comparison purposes, we have investigated also the photocyclization of the $P$-methoxy benzophospholene sulfide 6 (Scheme 8). ${ }^{[16]}$ The photocyclisation proceeds regioselectively, on the C4 carbon, leading to the [7]helicene 7a. It is worth noting that this compound has been isolated as a single diastereomer.

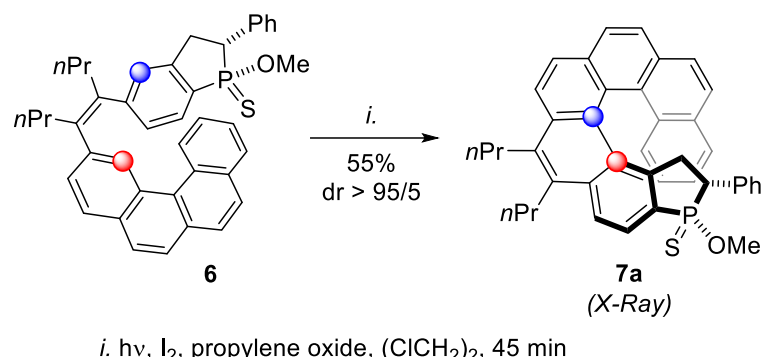

Scheme 8. Synthesis of the helical thiophosphinate $7 \mathrm{a}$ and acid $8 \mathrm{a}$ via regioand diastereoselective photocyclization of the thiophosphinate 6.

The molecular structure and stereochemistry of $7 \mathrm{a}$ have been ascertained by $\mathrm{X}$-ray crystallography (Figure 1). The compound displays relative $R_{P}, R_{C}$ and $P$-configurations at the three stereogenic units. Both the $\mathrm{MeO}$ group on phosphorus and the phenyl substituent on $\mathrm{C} 2$ are oriented toward the helical core.
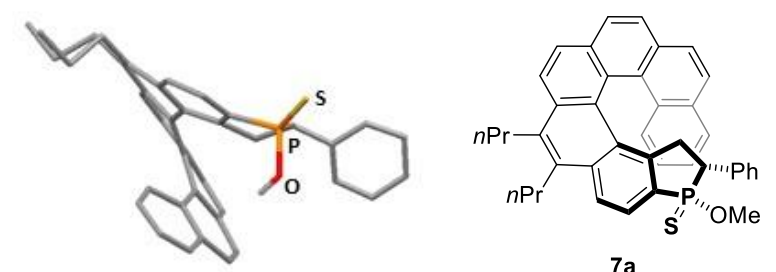

$7 a$

Figure 1. Crystal structure of the helical thiophosphinate 7a

Thus, isolation of $7 a$ from the photocyclisation experiment in Scheme 8 , together with the outcome of the cyclisation experiment in Scheme 7, demonstrate that the presence of the phospholene ring activates preferentially the C4-position of the fused phenyl ring toward oxidative photocyclisations, with respect to the C6-position. As said before, the opposite trend had been observed in the benzophosphole series, which led preferentially to cyclisation on C6 under analogous photochemical conditions.

Finally, the thiophosphinate $7 \mathrm{a}$ was converted quantitatively into the corresponding thiophosphinic acid $\mathbf{8 a}$ by removal of the methyl group by using DABCO. Compound $\mathbf{8 a}$ (Figure 2) is the first known helical thiophosphinic acids of the phosphahelicene series. As a complement, the diastereomeric acid $\mathbf{8} \mathbf{b}$ has been prepared also from the phosphinate $7^{\prime} \mathbf{b}$ via the thiophosphinate $\mathbf{7 b}$, as described in the Supporting information section.

In summary, the synthetic pathways above give access to two new series of phosphahelicenes with phosphinic and thiophosphinic ester and acid functions (Figure 2). The helical sequences of ortho-fused aromatic rings are terminated by either a meta-fused phosphole (3, 3') or an ortho-fused phospholene unit $(\mathbf{7}, \mathbf{8})$. In the two series, the phosphorus functions are located on the inner and external edges of the helix, respectively.

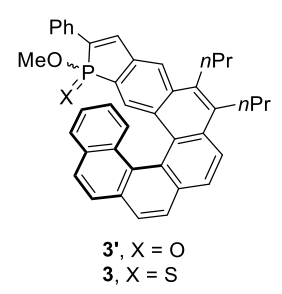

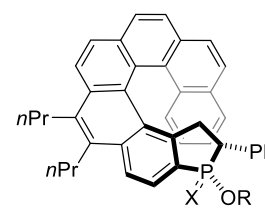

7'a, $R=M e, X=0$ 7a, $R=M e, X=S$ $8 \mathrm{a}, \mathrm{R}=\mathrm{H}, \mathrm{X}=\mathrm{S}$

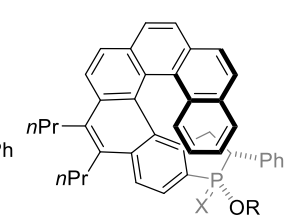
$8 \mathbf{b}, \mathrm{R}=\mathrm{H}, \mathrm{X}=\mathrm{S}$
$7 ' b, R=M e, X=0$ 7b, $R=M e, X=S$

Figure 2. Overview of the phosphinic/thiophosphinic acids and esters obtained in this work.

The synthetic procedures described in this paper have been carried out on racemic substrates. Nevertheless, enantiomerically pure samples of some of the new compounds have been obtained by SFC separation on CHIRALPAK columns (see SI for details). Examples include the thiophosphinates 7a $\left([\alpha]_{D}=+1300(\mathrm{c}=1\right.$, $\left.\left.\mathrm{CHCl}_{3}\right)\right)$ and $7 \mathrm{~b}\left([\alpha]_{\mathrm{D}}=+956\left(\mathrm{c}=0.5, \mathrm{CHCl}_{3}\right)\right)$. The corresponding thiophosphinic acids (+)-8a and (-)-8b have been obtained from enantioenriched $7 \mathbf{a}, \mathbf{b}$. Compounds with positive $[\alpha]_{\mathrm{D}}$ values are postulated to have a P-configured helical backbone. All these compounds display stereogenic phosphorus centres with defined configurations. ${ }^{[17]}$

\section{Computational studies on the oxidative photocyclisation reactions}

The computational studies above are intended to rationalize the divergent regioselectivity observed in the oxidative photocyclisations of phosphole and phospholene derivatives (see Scheme $4 b$ vs 8 ), via the free-valence number approach.

According to Coulson, the free valence index is defined as $F_{\text {, }}$ $=N_{\max }-N_{r}$, in which $N_{r}$ is the sum of the bond orders $(P)$ of all bonds joining atom $r$ and $N_{\max }$ is a constant that depends on the atom $r$ and its hybridization. ${ }^{[18]}$ For the types of carbons considered here, $N_{\max }=3+\sqrt{3}$ while $N_{r}$ is equal to 3 plus the sum of all $\pi$-bond orders between atom $r$ and the atoms it is bound to. ${ }^{[19]}$ The sum of the free valence numbers for the excited state, $\Sigma \mathrm{F}_{r, s}{ }^{*}$, for two positions $\mathrm{r}$ and $\mathrm{s}$ is usually given in a (somewhat confusing) notation as $\Sigma \mathrm{F}_{\mathrm{r}, \mathrm{s}}{ }^{*}=\mathrm{F}_{\mathrm{r}}{ }^{*}+\mathrm{F}_{\mathrm{s}}{ }^{*}=\left(\sqrt{3}-\Sigma \mathrm{P}_{\mathrm{r}}{ }^{*}\right)+(\sqrt{3}-$ $\left.\Sigma P_{s}{ }^{*}\right) \cdot{ }^{[20]}$ The general idea underlying the application of the free valence indices is that $F_{r}^{*}$ is large when the valence electrons are not too strongly tied up in covalent bonding. When two atoms $r$ and $s$ have both comparatively large $F_{r}{ }^{*}, F_{s}{ }^{*}$, then there is a larger tendency for the formation of local radicals, and bond formation between $r$ and $s$. Likewise, the difference of $\Sigma F_{r, s}{ }^{*}$ indices for different atom pairs may correlate with the regioselectivity of the reaction.

The actual values of the indices depend on the definition of the underlying bond orders. $N_{\max }$ and the sum of $\pi$-bond orders in the excited state, $\Sigma \mathrm{P}^{*}$, for a particular position were originally defined within the Hückel molecular orbital (HMO) theory. In HMO theory, the atomic orbital (AO) overlap is not explicitly treated, and a minimal basis set is used to describe the $\pi$ system only. We 


\section{WILEY-VCH}

\section{FULL PAPER}

therefore decided to perform free valence calculations for the excited states within a more modern framework in order to rationalize the cyclization regioselectivity for these helicene compounds. Namely, the $\pi$-bond orders entering the calculations of the free valence indices employed the Wiberg bond indices (WBIs) obtained for the lowest singlet excited state density matrix, from which the formal $\sigma$-bond orders, taken to be unity, were subtracted. For simple systems, the WBIs tend to correspond closely to the formal bond counts, and the TD-DFT excited state density should represent the selectivity of a system much better than the HMO approximation of the excited state and allows for a description of solvent effects by well-established models. We emphasize that the $\Sigma \mathrm{F}_{\mathrm{r}, \mathrm{s}}{ }^{*}$ based model is very simple, by design, and therefore we do not consider it sensitive enough such that it would, or should, make a difference whether the indices are calculated for the ground state (S0) or the excited state (S1) equilibrium structure, or at a conical intersection of SO and SO through which the photochemical reaction likely proceeds. In general, the photocyclization pathway is correlated to the largest value of $\Sigma \mathrm{F}_{\mathrm{r}, \mathrm{s}}{ }^{*}$.

Several reported stilbene-like compounds have been tested using our new procedure. The predictions are qualitatively consistent with previous $\mathrm{HMO}$ calculations regarding the viability and the regioselectivity of photodehydrocyclizations (see SI). Since we kept the $\sqrt{3}$ contribution to the free valence numbers the same as in past work, the criterion for when the photocyclization is viable or not is dependent on whether HMO bond orders or DFT / TDDFT WBIs are used. The latter are typically smaller for aromatic bonds than the former, leading to larger $\Sigma \mathrm{F}_{\mathrm{r}, \mathrm{s}}{ }^{*}$ values overall. The WBI-based calculations indicate that when $\Sigma \mathrm{F}_{\mathrm{r}, \mathrm{s}}{ }^{*}$ exceeds approximately 1.95 the photocyclization is viable, and the data for different carbon centers follow the same trend as the HMO-based calculations as far as the regioselectivity is concerned.

We next applied the TD-DFT/WBI-based free valence number scale to the compounds 2 and 6 (Table 1), by considering the relative free valence numbers of carbons $\mathrm{C} 4$ and $\mathrm{C} 6$. The position showing the largest free valence number, $F_{r}{ }^{*}$, corresponds to the most reactive site. In case of compound 2 , the $\Sigma \mathrm{F}_{1,6}{ }^{*}$ (1.991 in gas phase; 2.075 in solution) is larger than $\Sigma \mathrm{F}_{1,4^{*}}{ }^{*}(1.974$ in gas phase; 2.033 in solution), indicating that the formation of $\mathrm{C} 1-\mathrm{C} 6$ bond is favored, as observed in the experiments.
Table 1. Free valence numbers $\left(\Sigma F_{r, s}{ }^{*} ; F_{r}{ }^{*}\right)$ for compounds 2 and $\mathbf{6}^{a}$

\begin{tabular}{|c|c|c|c|c|}
\hline & Gas phase & & Solution & \\
\hline $\begin{array}{l}\text { Comp. } \\
\text { No. }\end{array}$ & $\Sigma \mathrm{F}_{\mathrm{r}, \mathrm{s}}{ }^{*}$ & $\mathrm{~F}_{\mathrm{r}}^{*}$ & $\Sigma \mathrm{F}_{\mathrm{r}, \mathrm{s}}{ }^{*}$ & $\mathrm{~F}_{\mathrm{r}}^{*}$ \\
\hline \multirow[t]{3}{*}{2} & $1,6=1.991$ & $1=0.986$ & $1,6=2.075$ & $1=1.044$ \\
\hline & $1,4=1.974$ & $4=0.988$ & $1,4=2.033$ & $4=0.989$ \\
\hline & & $6=1.005$ & & $6=1.031$ \\
\hline \multirow[t]{3}{*}{6} & $1,6=1.980$ & $1=1.058$ & $1,6=1.986$ & $1=1.063$ \\
\hline & $1,4=2.008$ & $4=0.950$ & $1,4=2.013$ & $4=0.950$ \\
\hline & & $6=0.922$ & & $6=0.923$ \\
\hline
\end{tabular}

a Computed free valence numbers in gas phase and in dichloroethane solution. Atoms involved (C1, C4 and C6) are marked in the figures. Dotted atom is the one preferentially involved in the photocyclisation reaction (experimental results).

On the other hand, for compound 6 , the $\Sigma F_{1,4}{ }^{*}$ (2.008 in gas phase 2.013 in solution) is higher than $\Sigma \mathrm{F}_{1,6^{*}}$ (1.980 in gas phase; 1.986 in solution), in agreement with the experiments showing that $\mathrm{C} 1$ C4 bond formation is favored.

The experimentally observed regioselectivity can therefore be rationalized with the free valence number model, as applied within a state-of-the-art electronic structure model for the excited states.

\section{Conclusions}

This work expands the family of phosphahelicenes to (thio)phosphinates and (thio)phosphinic acids which display configurationally stable stereogenic phosphorus centres. These new compounds represent one of the rare examples of $P$ stereogenic Brønsted acids reported in the literature.

This work also discloses the structurally new series of helicenes featuring terminal ortho-fused phospholene rings. The synthetic approach to this class of compounds takes advantage of a stereodefined benzophospholene building block, 5', that is obtained through diastereoselective hydrogenation of the corresponding 2-substituted P-methoxy-benzophosphole.

The Mallory's oxidative photocyclisation of diaryl-substituted olefins being the key step in the synthetic approaches above, we have highlighted a divergent regioselectivity of the photocyclisation reactions involving benzophospholes and benzophospholenes respectively. This behavior has been rationalized according to the free valence number model, by means of updated DFT calculations of the excited states of the olefinic substrates.

Overall this study expands the structural diversity of the helical phosphorus compounds obtained through the photocyclisation method.

Future work will be devoted to investigations of both the photophysical properties and organocatalytic applications of these new helical compounds. 
WILEY-VCH

\section{FULL PAPER}

\section{Acknowledgements}

Authors acknowledge financial support from the "Agence Nationale de la Recherche" (ANR) (HelPhos project: ANR-15CE29-0012-01) for grants to J.F. and C.D. The Centre National de la Recherche Scientifique (CNRS) and the "Indo-French Center for the Promotion of Advanced Research (IFCPAR/CEFIPRA, Project $\mathrm{N}^{\circ} 5505-2,2016$ ) are also acknowledged for financial support. We also acknowledge the Center for Computational Research (CCR), University at Buffalo for providing computational resources. The theoretical component of this study has been supported by Grant CHE-1560881 from the National Science Foundation (U.S.A.).

Keywords: phosphahelicenes $•$ thiophosphinic acids $\bullet P$ stereogenic $\cdot$ photocyclization $\cdot$ phospholenes

[1] a) Y. Shen, C.-F. Chen, Chem. Rev. 2012, 112, 1463-1535; b) M. Gingras, Chem. Soc. Rev. 2013, 42, 968-1006; c) M. Gingras, G. Félix, R. Peresutti, Chem. Soc. Rev. 2013, 42, 1007-1050; d) M. Gingras, Chem. Soc. Rev. 2013, 42, 1051-1095; e) M. Hasan, V. Borovkov, Symmetry 2018, 10 doi:10.3390/sym10010010. f) K. Dhbaibi, L. Favereau, J. Crassous, Chem. Rev. 2019,119, 8846-8953.

[2] a) M. J. Narcis, N. Takenaka, Eur. J. Org. Chem. 2014, 21-34; b) P. Aillard A. Voituriez, A. Marinetti, Dalton Trans 2014, 43, 15263-15278; c) J. OuYang, J. Crassous, Coord. Chem. Rev. 2018, 376, 533-547.

[3] C. S. Demmer, A. Voituriez, A. Marinetti, C.R. Chimie 2017, 20, 860-879.

[4] a) N. Fukawa, T. Osaka, K. Noguchi, K. Tanaka, Org. Lett. 2010, 12, 1324 1327 ; b) K. Nakano, H. Oyama, Y. Nishimura, S. Nakasako, K. Nozaki, Angew. Chem. Int. Ed. 2012, 51, 695-699; c) S. Hashimoto, S. Nakatsuka, M. Nakamura, T. Hatakeyama, Angew. Chem. Int. Ed. 2014, 53, 1407414076; d) S. Nishigaki, K. Murayma, Y. Shibata, K. Tanaka, Mater. Chem. Front. 2018, 2, 585-590.

[5] a) K. Yavari, S. Moussa, B. Ben Hassine, P. Retailleau, A. Voituriez, A. Marinetti, Angew. Chem. Int. Ed. 2012, 51, 6748-6752; b) K. Yavari, P. Retailleau, A. Voituriez, A. Marinetti, Chem. Eur. J. 2013, 19, 9939-9947 c) P. Aillard, A. Voituriez, D. Dova, S. Cauteruccio, E. Licandro, A. Marinetti, Chem. Eur. J. 2014, 20, 12373-12376; d) P. Aillard, P. Retailleau, A Voituriez, A. Marinetti, Chem. Commun. 2014, 50, 2199-2201; e) P. Aillard, P. Retailleau, A. Voituriez, A. Marinetti, Chem. Eur. J. 2015, 21, 11989 11993; f) M. S. Rahman, N. Yoshikai, Org. Lett. 2019, 21, 3232-3236.

[6] C. S. Demmer, P. Aillard, J. Febvay, P. Retailleau, A. Voituriez, A. Marinetti, ChemPhotoChem 2017, 1, 535-538.

[7] Y. Sawada, S. Furumi, A. Takai, M. Takeuchi, K. Noguchi, K. Tanaka, J. Am. Chem. Soc. 2012, 134, 4080-4083.

[8] a) K. Yavari, P. Aillard, Y. Zhang, F. Nuter, P. Retailleau, A. Voituriez, A. Marinetti, Angew. Chem. Int. Ed. 2014, 53, 861-865; b) M. Gicquel, Y Zhang, P. Aillard, P. Retailleau, A. Voituriez, A. Marinetti, Angew. Chem. Int. Ed. 2015, 54, 5470-5473.

[9] K. Yavari, W. Delaunay, N. De Rycke, T. Reynaldo, P. Aillard, M. SrebroHooper, V. Y. Chang, G. Muller, D. Tondelier, B. Geoffroy, A. Voituriez, A. Marinetti, M. Hissler, J. Crassous, Chem. Eur. J. 2019 10.1002/chem.201806140

[10] a) F. B. Mallory, C. S. Wood, J. T. Gordon, L. C. Lindquist, M. L. Savitz, J. Am. Chem. Soc. 1962, 84, 4361-4362; b) F. B. Mallory, C. W. Mallory, Org React. 1984, 30, 1; c) R. H. Martin, Angew. Chem. Int. Ed. 1974, 13, 649660.

[11] D. J. Carr, J. S. Kudavalli, K. S. Dunne, H. Müller-Bunz, D. G. Gilheany, J. Org. Chem. 2013, 78, 10500-10505.

[12] a) N. P. Kenny, K. V. Rajendran, E. V. Jennings, D. G. Gilheany, Chem. Eur. J. 2013, 19, 14210-14214; b) K. M.-H. Lim, T. Hayashi, J. Am. Chem. Soc. 2017, 139, 8122-8125

[13] a) L. Hintermann, M. Schmitz, O. V. Maltsev, P. Naumov, Synthesis 2013, 45, 308-325; b) G. Hum, K. Wooler, J. Lee, S. D. Taylor, Can. J. Chem. 2000, 78, 642-655.

[14] Sulfuration is known to take place with retention of the phosphorus configuration: a) A. M. Polozov, E. E. Cremer, P. E. Fanwick, Can. J. Chem. 1999, 77, 1274-1280; b) T. Ozturk, E. Ertas, O. Mert, Chem. Rev. 2007, 107, 5210-5278.

[15] a) M. Scholz, M. Mühlstädt, F. Dietz, Tetrahedron Lett. 1967, 665-668; b) W. H. Laarhoven, T. J. H. M. Cuppen, R. J. F. Nivard, Recl. Trav. Chim Pays-Bas 1968, 87, 687-698; c) W. H. Laarhoven, T. J. H. M. Cuppen, R. J. F. Nivard, Tetrahedron 1970, 26, 4865-4881; d) W. H. Laarhoven, Recl. Trav. Chim. Pays-Bas 1983, 102, 185-204; e) A. D. Dinga Wonanke, J. L. Ferguson, C. M. Fitchett, D. L. Crittenden, Chem. Asian J. 2019, 14, 1293 1303; f) J. Weber, E. L. Clennan, J. Org. Chem. 2018, 84, 817-830.
[16] Sulfide $\mathbf{6}$ is obtained from $\mathbf{6}^{\prime}$ by sulfuration with the Lawesson reagent as described in the S.I.

[17] For selected examples of P-stereogenic phosphinates, phosphinic acids or amides, see: a) Y. Maekawa, K. Kuwabara, A. Sugiyama, K. Iwata, T. Maruyama, T. Murai, Chem. Lett. 2017, 46, 1068-1071; b) S. Liu, Y. Li, Q. Yu, Z. Miao, J. Org. Chem. 2017, 82, 2481; c) R. Soengas, E. Belmonte Sanchez, M-J. Iglesias, F.L. Ortiz, ACS Omega 2018, 3, 5116-5124; d) JL. Montchamp, Pure Appl. Chem. 2019, 91, 113-120 and references therein

[18] a) C. A. Coulson, Faraday Discuss. 1947, 2, 9-18; b) C. A. Coulson, J. Chim. Phys.-Chim.Biol, 1948, 45, U247-248

[19] a) J. D. Roberts, A. Streitwieser, Jr., C. M. Regan, J. Am. Chem. Soc. 1952 74, 4579-4582; b) H. H. Greenwood, Trans. Faraday Soc., 1952, 48, 677679

[20] N. Hoffmann, J. Photochem. Photobiol., C, 2014, 19, 1-19 
WILEY-VCH

\section{FULL PAPER}

\section{Divergent photocyclisations}

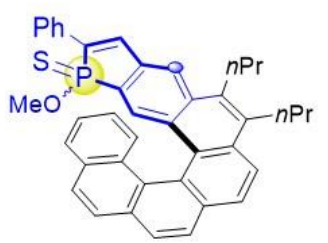

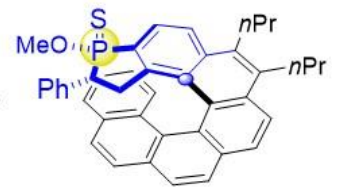

J. Febvay, C. Demmer, P. Retailleau, J. Crassous, L. Abella, J. Autschbach, ${ }^{*}$ A. Voituriez, ${ }^{*}$ A. Marinetti*

Page No. - Page No.

Phosphahelicenes with (thio)phosphinic acid and ester functions via the oxidative photocyclisation approach

Phosphahelicenes with thiophosphinite functions are produced via the Mallory oxidative photocyclisation approach. Phospholes and phospholenes activate different positions of the substrates, thus leading to helicenes terminated by a meta-fused phosphole and an ortho-fused phospholene ring respectively. 